\title{
XVII.
}

\section{Ein Fall von angeborner knöcherner Ankylose des linken Kiefergelenks.}

\author{
Von Dr. med. B. Sólger, \\ Assistenten am anatomischen Institute zu Heitiellerg.
}

(Hierzu Taf. IX.)

Die folgenden Zeilen entbalten die Beschreibung einer Missbildung, zu welcher sich in der Literatur kaum ein Seitenstïck finden dürfte. Wenigstens erwähnen Rokitansky (Lehrbuch der path. Anat. III. Auflage 1855.) und Förster (Missbildungen) derselben nicht, und auch in Zeitschriften konnte eine derartige Veröffentlichung nicht aufgefunden werden.

Es handelt sich um einen Fall von angeborner knöcherner Ankylose des linken Kiefergelenkes. Der Schädel ist der eines Erwachsenen: die Kranznaht ist vollständig, die Pfeilnaht grösstentheils verstrichen, der dritte Malzahn des Oberkiefers linkerseits vorhanden, rechterseits aus der Alveole gefallen. Hinterhauptsbein und rechtes Schläfenbein sind verloren gegangen; das der linken Seite wird bei Beschreibung des Unterkieferstückes genauer besprochen werden. Das rechte Scbeitelbein ist in der Gegend des Taher parietale mehr nach aussen gewölbt als linkerseits, wodurch das Schädeldach bei Betrachtung von oben unsymmetrisch nad rechts geräumiger erscheint. Auf derselben Seite ist der Processus palatinus des Oberkiefers und namentlich die Pars horizontalis des Gaumenbeins entsprechend dem tieferen Ausschnitt der rechten Hälfte der Apertura pyriformis nach unten vorgewölbt. Die Lamina externa des rechten Processus pterygoideus des Keilbeins unterscheidet sich, als ein mächtiges, an der Aussenfläche mit rauhen Leisten besetztes Knochenblatt auffallend von der nur wenig entwickelten äusseren Lamelle der linken Seite, wo dagegen die Fossa pteroidea geräumiger erscheint. - Die Verbältnisse der Oberkieferzähne und ihrer Alveolen verdienen genanere Beschreibung. Von den Schneidezahnen, die jetzt feblen, sind währead des Lebens nur die zwei äusseren vorhanden gewesen, wie die entsprechenden Alveolen beweisen. Zahnfächer für die beiden inneren sind nicht zu erkennen und stellt der Alveolarfortsatz hier nur eine dünne Knochenkante dar, binter der eine alveolenähnliche Höhle auftritt: das erweiterte Foramen incisivum. Von den übrigen Zäbnen ist rechterseits nur der erste Backzahn in seiner Alreole geblieben, dessen Kanfäche in ihrer hinteren Hälfte wie durch Caries zerstört, vorn dagegen glatt abgeschliffen erscheint. Die Alveolen der übrigen Zähne dieser Seite sind normal. Die linke Seite trägt 
noch drei Zähne: die beiden Backzähne und den Weisheitszahn. Das Fehlen der beiden übrigen Malzähne erklärt sich leicht aus der Form der zugehörigen diveolen, die nur einfache, mehr oder weniger ausgesprochene Vertiefungen mit glatter Wandung darstellen.

Wenden wir uns nun zur Beschreibung der durch Ankylose verbundenen Knochen, des linken Schläfenbeins und des entsprechenden Theils des Voterkiefers. Pyramyde und Warzentheil bieten somit sicbtbar keine ungewöhnlichen Verhältaisse dar, ebensowenig die Fissura petro-lympanica una die Fissura petroso-squamosa; die sbweichungen rom Normalen beschrünken sich anf die untere Partie des Schuppentheils.

Die ganze untere Fläche des Schuppentheils, unmittelbar nach vorn von der zuerst genannten Fissur bis zu der an das Keilbein stassenden Naht des Knochens wird von einer mächtigen, auf dem Querschnitt ovalen Knochensäule eingenommen, die zunächst nach aussen vorn und etwas nach unten verläuft, dann in stumpfen Winkel geknickt und gleichzeitig in der Richtung vón aussen nach innen zusammengedrückt, nach unten und vorn sich wendet. Während nun eine melir oder weniger ausgesprochene Furche an der hinteren und inneren Fläche der Basis des Knochencylinders die Grenze beider Knochen markirt, trennt eine anssen und vorn bogenförmig verlaufende Nabt einen dem Scluppentheil angehörigen kleineren Theil (Fig. I a) von dem den Unterkiefer repräsentirenden grösseren Theil (b) und einem mit seinem rorderen Ende dem Jochbein sich anlagernden Knochen $(x)$, der an ein bei Säugethieren freilich nicbt vorkommendes 0s quadratum erinnert. Sein binteres breites Ende erscheint wiederum wó der äusseren Fläche des Unterkiefers nach unten convergirende Sehenkel einer zackigen Naht abgegrenzt (s. Fig. 1). Das mit dem oben beschriebenen, dem Schuppentheil angehörigen Knochenzapfen a und dem eingescbalteten Stäck e verwachsene Ende des Unterkiefers entspricht dem Processus condyloideus; der Processus coronoideus wird durch einen schmalen, dreikantigen, oben scharf ausgezogenen Fortsatz repräsentirt, der durch eine Spalte, die verengte halbmondförmige Incisur, von dem hinter ihm liegenden massigen Theil getreant wird.

Die Betrachtung des ïbrigen Theils des Unterkiefer-Rudiments, das etwa $8 \mathrm{~cm}$. in der Länge misst und in der Gegend des ersteren linken Malzahnes abgebrochen ist, lässt vor Allem eine scharfe Grenze zwischen einem horizontalen und einem aufsteigenden Ast vermissen. Es berubt dieses theils daranf, dass die Axen beider in sehr stumpfen Winkel auf einander stossen, theils darauf, dass der dem Körper entsprechende Theit in seinem vorderen Rande weder Zähne noch Alveolen trägt, sondern statt dessen nur zwei Knochendornen aufweist. Darnach ist, wie die Betrachtung der inneren Fläche lehrt, ein Zabn verhanden, der mit einem Theil der Krone und der konischen Wurzel frei zu Tage liegt, sonst aber von Knochenmasse umschlossen ist (Fig. 3). Er wird als Malzahn aufufassen sein. Die innere Fläche zeigt noch das Foramen maxillare internum, den Sulcus mylohyoideus, an der äusseren Fläche ist das einige Linien rom unteren Rande des Unterkieferstücks befindliche Foramen mentale za erwähnen. Eine von da nach oben gezogene Senkrechte triff den zweiten oberen Malzahn, fällt also ungewöhnlich weit nach hinten, was sich aus der geringen Entwickelung und aus der vorwiegend nach unten 


\section{2}

gerichteten Längsaxe des beschriebenen Knochens erklärt. Eine weit auffallendere Lagerungsabweichung desselben zum Oberkiefer ergiebt sich bei Betracbtung von unten (Fig. 3) : der Unterkiefer beschreibt nehmlich einen grösseren Bogen als die Peripherie des Oberkiefers, so dass der äussere Alveolarrand des letzteren noch weiter medianwärts zu liegen kommt als die innere Fläche des Unterkieferstücks.

So leicht bei derartigen offen zu Tage liegenden Verbältnissen die Beobachlung, so schwierig die Reflexion. Es bandelt sich hier um eine Missbildung im Bereich von nicht mehr als zwei Knochen, der Unterkiefer einerseits, des - hier selbständig gedachten Schuppentheils des Schläfenbeins andererseits, Nun entwickelt sich die Schuppe bekanntlich getrennt vom knorpelig vorgebildeten os petroso-mastoideum, gleichzeitig mil dem Annulus tympanicus als Deck- oder Belegknochen des Schädels jedenfalls schon während des dritten Monats. Das Auftreten eines fadenförmigen (filiformis) Jochfortsatzes setat Senf( ${ }^{1}$ ) in die 11. Woche. Früher schon, etwa in der 6. - 7. Woche hat die Entwickelung des Unterkiefers begonnen, der ohne knorpelig vorgebildet zu sein, von der Aussenseite des Meckel'schen Fortsatzes auftritt, zu dem er "genau in demselben Verhällnisse stebt, wie die Deckknochen am Schädel zum Primordial-cranium" (Koellicker, Vorles. über EntwickelungsGesch., S. 217). Wir haben es wohl in unserem Fall mit einer frühzeitigen knöchernen Verschmelzung zweier nach dem Typus Deckknochen, d. h. "aus einer morphologisch nicht bestimmten Grundlage" (Koellicker) sich entwickelnden Knochen zu thun, deren Grenze nur zum Theil durch die oben hescbriebene Naht markirt ist. Denken wir uns, gestützt auf die Zeitangaben der Entwickelung des Unterkiefers (6.-7. Woche) and des Processus zygomaticus (11. Woche) den Prozess in der Gegend des Rielergelenkes schon weiter vorgeschrilten, bevor es zur Bildung des Processus zygomalicus des Schläfenbeins gekommen ist, so würde sich daraus das Verhalter des oben beschriebenen als Jochfortsatz aufzufassenden Schalt knochens $x$ erklären. Freilich würde damit die Existenz eines selb ständig im Processus zygomaticus auftretenden Ossifications-Punkt€ vorausgesetzt, der kaum direct beobachtet worden ist. Die Annahr eines sehr frübzeitigen Auftretens des Verschmelzungsprozess

1) c. F. Senff, Nonaula de incremento ossium embryonum in primis gre ditatis temporibus. Halae 1802 . 
steht mit dem Vorhandensein des aus naheliegenden Gründen verkümmerten Processus coronoideus nicht im Widerspruch. Ueber die Verhältnisse der rechten Unterkieferhälfte und des entsprechenden Kiefergelenkes während des Lebens lassen sich nur Vermuthungen hegen; die starke Entwickelung der Lamina externa des Flügelfortsatzes dieser Seite, die abgeschliffene Oberfläche des ersten oberen Backzabnes, der hier gut entwickelte Alveolarrand lassen sich als Momente, die für die Ausübung der Kaufunction sprechen, verwerthen. $O b$ erst ein operativer Eingriff das Kauen auf dieser Seite ermöglichte, oder ob etwa bei normalem rechten Kiefergelenk beide Unterkieferhälften getrennt persistirten und so eine isolirte Beweglichkeit dieser Hälfte möglich war, lässt sich natürlich nicht mehr entscheiden. ${ }^{1}$ )

Der bescliriebene Schädel hefindet sich in der anatomischen Sammlung zu Jena und wurde durch Herrn Hofrath Gegenba a r mir bereitwilligst zur Verfügung gestellt, dem ich hiermit für die freundliche Unterstützung dieser kleinen Arbeit den gebührenden Dank ausspreche.

1) Vielleicht liesse sich auch das Auftreten eines rudimentären Zabns in dem ankylosirten Unterkieferstück gerade an der engsten Stelle zwischen demselben und der linken Oberkieferbälfte darauf zurückführen, dass hier die Berührung der Theile mit den eingeführten Ingestis am intensivsten sein musste. 